ARTIGO ORIGINAL

\title{
Perfil da aptidão física em crianças e adolescentes praticantes de mini tênis em Jacarezinho (PR)
}

\section{Profile of physical fitness in children and adolescents practicing mini tennis in Jacarezinho, PR, Brazil}

\author{
Flávia Évelin Bandeira Lima ${ }^{1}$, Walcir Ferreira Lima ${ }^{1}$, Mariane Aparecida Coco ${ }^{1}$, \\ Fellipe Bandeira Lima², Silvia Bandeira da Silva Lima ${ }^{1}$ \\ ${ }^{1}$ Universidade Estadual do Norte do Paraná (UENP), Jacarezinho/PR, Brasil \\ ${ }^{2}$ Centro Universitário Campos de Andrade (Uniandrade), Curitiba/PR, Brasil
}

\author{
HISTÓRICO DO ARTIGO \\ Recebido: 17 abril 2020 \\ Revisado: 16 junho 2020 \\ Aprovado: 18 junho 2020
}

\section{PALAVRAS-CHAVE:}

Aptidão física; Esportes com raquetes; Tênis.

\section{KEYWORDS:}

Physical fitness; Racquet sports; Tennis.

\section{RESUMO}

INTRODUÇÃO: Dentre as modalidades de esporte, cada uma tem suas exigências particulares e requerem características e habilidades especificas, que delimitam o comportamento motor de uma criança. OBJETIVO: Identificar e comparar o perfil da aptidão física relacionada ao desempenho motor e à saúde em estudantes de 6 a 13 anos de idade praticantes de mini tênis.

MÉTODOS: Pesquisa transversal, realizada com 132 estudantes (72 meninas e 60 meninos), praticantes de mini tênis. Avaliou-se a massa corporal $(\mathrm{kg})$, estatura $(\mathrm{cm})$ e circunferência da cintura $(\mathrm{cm})$. Em seguida, aplicou-se os testes motores da bateria do Proesp (2016): índice de massa corporal (IMC), relação cintura/estatura (RCE), teste de sentar e alcançar, teste de corrida/caminhada dos 6 minutos, abdominal em 1 minuto, arremesso da medicineball, salto horizontal, teste de agilidade do quadrado e corrida de 20 metros. Após, os dados foram tratados por meio do SPSS, versão 26.0. Utilizou-se os testes de U de Mann-Whitney, Kolmogorov-Smirnov e Qui-quadrado (x2).

RESULTADOS: Os resultados não foram satisfatórios para IMC, flexibilidade (sexo masculino), agilidade e velocidade. Entretanto, nos testes de força explosiva de membros inferiores e força explosiva de membros superiores, ambos os sexos atenderam aos critérios de desempenho.

CONCLUSÃO: Este estudo encontrou diferenças entre os sexos nas variáveis de flexibilidade e força-resistência abdominal. Após um ano de prática de mini tênis, a aptidão física dos escolares avaliados está abaixo do esperado, visto que, o IMC está elevado para a idade e o desempenho nos testes de agilidade e velocidade (essenciais para o mini tênis) não foi positivo.

\section{ABSTRACT}

BACKGROUND: Among the sports modalities, each one has its particular requirements and require specific characteristics and skills, which delimit a child's motor behavior.

OBJECTIVE: To identify and compare the profile of physical fitness related to motor performance and health in students from 6 to 13 years old who practice mini tennis.

METHODS: Cross-sectional survey, carried out with 132 students (72 girls and 60 boys), who practice mini tennis. Body mass $(\mathrm{kg})$, height $(\mathrm{cm})$ and waist circumference $(\mathrm{cm})$ were evaluated. Then, the motor tests of the Proesp battery (2016) were applied: body mass index (BMI), waist/height ratio (WHR), sit and reach test, 6-minute running/walking test, one-minute sit-up test, medicineball pitch, standing long jump test, square agility test and 20 meter dash run test. Afterwards, the data were treated using SPSS, version 26.0. MannWhitney $\mathrm{U}$ tests (median), Kolmogorov-Smirnov and Chi-square $(\chi 2)$ were used.

RESULTS: The results were not satisfactory for BMI, flexibility (male), agility and speed. However, in the tests of explosive strength of lower limbs and explosive strength of upper limbs, both sexes met the performance criteria.

CONCLUSION: This study found differences between the sexes in the variables of flexibility and abdominal strength-resistance. After a year of practicing mini tennis, the physical fitness of the students evaluated is below expectations, since the BMI is high for age and the performance in the agility and speed tests (essential for mini tennis) was not positive. 


\section{INTRODUÇÃO}

Uma série de alterações ocorrem durante o processo de desenvolvimento motor, no qual aspectos do crescimento físico, da maturação, do desenvolvimento da aptidão física, da atividade física, da idade e da experiência estão associados (SILVA et al., 2017). A aptidão física se divide em dois parâmetros e habilidades de acordo com a necessidade de cada indivíduo, sendo aptidão física relacionada a saúde (ApFRS) e ao desempenho (ApFRD). Compreendem, respectivamente, as capacidades físicas: força/resistência muscular, resistência cardiorrespiratória, flexibilidade, velocidade, agilidade, potência (ou força explosiva), coordenação e equilíbrio (VENÂNCIO et al., 2018).

Nessa perspectiva, alguns estudos brasileiros têm demonstrado que a ApFRD dos jovens não atinge níveis adequados, e que cerca de $40 \%$ dos jovens têm desempenho ruim ou regular (MELLO et al., 2015; DUMITH et al., 2010). Gaya et al. (2002) indicam a nível populacional, que é em torno de $2 \%$ a possibilidade esperada de adolescentes que irão atingir o desempenho excelente para o talento para a prática esportiva.

Dentre as modalidades de esporte, cada uma tem suas exigências particulares e requerem características e habilidades especificas, que delimitam o comportamento motor de uma criança (SILVA; CONTENÇAS; MARQUES, 2017). Esportes com raquetes demonstram que as habilidades adquiridas nessa modalidade fazem uma relação importante com os movimentos fundamentais e com as habilidades especializadas (GALLAHUE; OZMUN; GOODWAY, 2013). Chiminazzo e Azzi (2005), afirmam que o jogo de tênis, por ser um esporte individual, precisa de um grande esforço por parte de seus praticantes. O jogador deve ter consciência de que se ele não usufruir do jogo, com certeza o seu parceiro também não usufruirá. Este embasamento deverá ser adquirido pelo processo educativo. É fundamental jogar com o outro e não contra o outro.

Encontra-se no mini tênis, a possibilidade de estimular o aluno a abandonar o processo de aprendizagem passivo, criando, interagindo e apoderando-se do jogo dentro do potencial das suas possibilidades. Não se trata de uma prática esportiva excludente que limita o jogo em razão das intempéries, do espaço ou do número de jogadores, pelo contrário, trata-se de uma prática esportiva que apresenta poucos obstáculos para sua execução como um jogo ou uma brincadeira (COSTA, 2019).

O minitênis é jogado em uma quadra pequena e simples, muito parecida com uma quadra de Badminton, pode ser construída em qualquer superfície plana e jogado com qualquer tipo de raquete (que não seja pesada ou com a empunhadura grande), até mesmo as raquetes de madeira, plástico ou alumínio. É um jogo divertido e recreativo, adaptado do tênis de campo, servindo como introdução ao mesmo (ITF, 1993). Brustolin (1995) define o mini tênis como "o tênis de campo em menores proporções", ou seja, quadras menores, raquetes menores, redes mais baixas, bolas mais leves, e regras mais simples. A base do aprendizado está no controle de bola e nos jogos.

Recomenda-se, cada vez mais, o mini tênis como jogo desportivo a ser desenvolvido, em virtude de sua contribuição no progresso motor e no desenvolvimento de novas habilidades das crianças em conjunto com o desenvolvimento das capacidades biomotoras. E, por contribuir efetivamente na formação in- tegral das crianças, aspectos esse que o jogo pode proporcionar.

Diante do exposto, uma vez que, a aptidão física voltada para a modalidade de tênis de campo é pouco evidenciada na literatura, pesquisas são necessárias para colaborar com o planejamento e desenvolvimento de ações para promoção da saúde no âmbito escolar e extraescolar. Assim, buscou-se identificar e comparar o perfil da aptidão física relacionada ao desempenho motor e à saúde em estudantes de 6 a 13 anos de idade praticantes de mini tênis.

\section{MÉTODOS}

Trata-se de um estudo com delineamento metodológico transversal, realizado com crianças de 6 a 13 anos de idade, participantes de um programa de extensão universitária, intitulado "Programa mini tênis para crianças e adolescentes", realizado pela Universidade Estadual do Norte do Paraná (Jacarezinho/ $\mathrm{PR}$ ) em parceria com a Associação Ícaro Marcolin (Curitiba/PR). O estudo foi aprovado pelo Comitê de Ética em Pesquisa da Universidade Estadual do Norte do Paraná (Parecer no 3.083.384; CAEE: 91513116.6.0000.0108).

Participaram do estudo, 132 escolares (Meninas: $n=72 ;$ Meninos: $n=60$ ), com idades entre 6 e 13 anos, todos praticantes de mini tênis. $O$ volume de treinamento foi de 2 horas semanais, exatamente as terças e quintas-feiras, em horário de contraturno escolar, sendo divididos em três turmas: das 09:00 as 10:00 horas da manhã e das $15: 30$ as 16:30 horas da tarde, para os escolares de 6 a 8 anos de idade. E, das 14:00 as 15:00 horas da tarde para os estudantes de 9 a 13 anos de idade.

Como critérios de inclusão, foram selecionados apenas aqueles com idades de 6 a 13 anos, que participavam dos treinamentos há pelo menos um ano, com uma frequência mínima de $75 \%$, que retornaram com o TCLE assinado junto com seus pais ou responsável e que participaram de todos os testes referentes ao estudo. São motivos de exclusão os que não se enquadrarem nas referências acima.

Todos os participantes foram informados dos propósitos, riscos e benefícios do estudo e que poderiam, sem constrangimento, deixar de participar da pesquisa quando desejassem. Os pais foram devidamente comunicados e informações sobre a importância, os objetivos e a metodologia do estudo, receberam o Termo de Consentimento Livre Esclarecido para a autorização dos participantes na pesquisa.

O protocolo de teste utilizado foi do Projeto Esporte Brasil (PROESP-Br, 2016). Obedeceu-se a seguinte sequência: Massa corporal $(\mathrm{kg})$ : balança digital; Estatura $(\mathrm{cm})$ : medida em estadiômetro portátil; Índice de Massa Corporal (IMC): massa (kg)/ estatura $\left(\mathrm{m}^{2}\right)$; Relação Cintura Estatura (RCE): perímetro da cintura $(\mathrm{cm})$ /estatura $(\mathrm{cm})$; Teste de sentar-e-alcançar (flexibilidade) (cm); Teste de corrida/caminhada dos 6 minutos (resistência geral) (metros); Abdominal (força-resistência abdominal) (quantidade); Arremesso da medicineball (força explosiva de membros superiores - FMS) (cm); Salto horizontal (força explosiva de membros inferiores - FMI) (cm); Teste do quadrado (agilidade) (segundos e centésimos de segundos - duas casas após a vírgula); Teste de corrida de 20 metros (velocidade) (segundos e centésimos de segundos - duas casas após a vírgula).

Após a coleta os dados foram tratados por meio do pacote computadorizado Statistical Package for the Social Science 
(SPSS), versão 26.0. Para a análise das variáveis numéricas recorreu-se aos procedimentos da estatística descritiva (frequência absoluta e relativa), posteriormente, para identificação de eventuais diferenças, ao teste $\mathrm{U}$ de Mann-Whitney (mediana), segundo resultados do teste de normalidade Kolmogorov-Smirnov.

As variáveis categóricas (proporção de frequência de acordo com os pontos de corte considerados) foram analisadas mediante tabelas cruzadas, envolvendo testes de Qui-quadrado

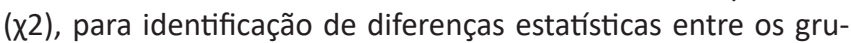
pos (comparações múltiplas ajustadas pelo método Bonferroni. Os valores de $p<0,05$ foram considerados estatisticamente significativos para todas as análises.

\section{RESULTADOS}

A amostra foi composta por 132 crianças e adolescentes (Meninas: $n=72 ; 54,5 \%$; Meninos: $n=60 ; 45,5 \%$ ). Observa-se que a maioria do sexo feminino compreende as idades de 6 e 7 anos, já o sexo masculino, 6 e 8 anos (Figura 1).

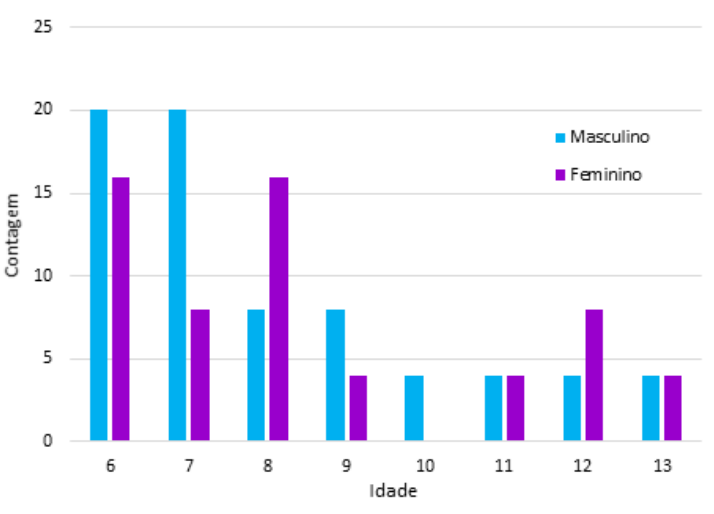

Figura 1. Contagem de escolares praticantes de mini tênis, em relação a idade, em Jacarezinho $(\mathrm{Pr})$.

A proporção de obesidade geral, segundo o IMC, indicou que a maioria dos participantes são classificados na zona de risco à saúde $(78,8 \%)$, de acordo com o protocolo de avaliação do Proesp (2016). E, para a RCE, 81,8\% dos estudantes estão na zona saudável, com melhores resultados para as meninas (Tabela 1).

No teste de flexibilidade, nota-se uma proporção elevada de meninas e meninos no grupo de risco à saúde (51,5\%), ao analisar por sexo, os meninos apresentaram ter menor flexibilidade que seus pares $(80,0 \%$ vs. $27,8 \% ; p<0,001)$. Nos testes de força/resistência abdominal, identifica-se $22,2 \%$ G-Fem vs. $53,3 \%$ G-Mas classificados na zona de risco. Por outro lado, para a resistência geral, a maioria, tanto o sexo feminino quanto o sexo masculino encontram-se dentro da zona saudável à saúde (83,3\% vs. $80,0 \%$, respectivamente).

Nas variáveis de desempenho motor identifica-se que não houve diferenças estatisticamente significativas entre o G-Fem e o G-Masc apenas no indicador de resistência geral $(p=0,621)$. Ainda, observa-se que houve maior proporção $(60,6 \%$ igualmente) de indivíduos que alcançaram resultado positivo (>bom) apenas na FMS e FMI. Por fim, no teste de velocidade, as me- ninas se destacaram com os melhores resultados (38,9\% bom). Tabela 1. Distribuição da proporção (\%) de praticantes de mini tênis na avaliação da aptidão física em relação ao sexo.

\begin{tabular}{|c|c|c|c|c|c|}
\hline \multirow[t]{2}{*}{ Variáveis } & \multirow[t]{2}{*}{ Categorias } & $\begin{array}{l}\text { G-Fem } \\
(n=72)\end{array}$ & $\begin{array}{l}\text { G-Masc } \\
(n=60)\end{array}$ & $\begin{array}{c}\text { TOTAL } \\
(n=132)\end{array}$ & \multirow[t]{2}{*}{ p-valor } \\
\hline & & $f(\%)$ & $f(\%)$ & $f(\%)$ & \\
\hline \multirow{2}{*}{$\begin{array}{c}\text { IMC } \\
(\mathrm{kg} / \mathrm{m} 2)\end{array}$} & Saudável & $20(27,8)$ & $8(13,3)$ & $28(21,2)$ & \multirow{2}{*}{$0,043^{*}$} \\
\hline & Risco à Saúde & $52(72,2)$ & $52(86,7)$ & $104(78,8)$ & \\
\hline \multirow{2}{*}{$\begin{array}{l}\text { RCE } \\
(\mathrm{cm})\end{array}$} & Saudável & $68(94,4)$ & $40(66,7)$ & $108(81,8)$ & \multirow{2}{*}{$<0,001^{*}$} \\
\hline & Risco à Saúde & $4(5,6)$ & $20(33,3)$ & $24(18,2)$ & \\
\hline \multirow{2}{*}{$\begin{array}{l}\text { Sentar-e-al- } \\
\text { cançar } \\
\text { (cm) }\end{array}$} & Saudável & $52(72,2)$ & $12(20)$ & $64(48,5)$ & \multirow{2}{*}{$<0,001^{*}$} \\
\hline & Risco à Saúde & $20(27,8)$ & $48(80)$ & $68(51,5)$ & \\
\hline \multirow{2}{*}{$\begin{array}{l}\text { Resistência } \\
\text { Geral } \\
\text { (m) }\end{array}$} & Saudável & $60(83,3)$ & $48(80)$ & $108(81,8)$ & \multirow[b]{2}{*}{0,621} \\
\hline & Risco à Saúde & $12(16,7)$ & $12(20)$ & $24(18,2)$ & \\
\hline \multirow{2}{*}{$\begin{array}{l}\text { Abdominal } \\
\text { (rep.) }\end{array}$} & Saudável & $56(77,8)$ & $28(46,7)$ & $84(63,6)$ & \multirow{2}{*}{$<0,001^{*}$} \\
\hline & Risco à Saúde & $16(22,2)$ & $32(53,3)$ & $48(36,4)$ & \\
\hline \multirow{5}{*}{$\begin{array}{l}\mathrm{FMl} \\
(\mathrm{cm})\end{array}$} & Fraco & $20(27,8)$ & $28(46,7)$ & $48(36,4)$ & \multirow{5}{*}{$<0,001^{*}$} \\
\hline & Razoável & $4(5,6)$ & - & $4(3)$ & \\
\hline & Bom & $12(16,7)$ & $20(33,3)$ & $32(24,2)$ & \\
\hline & Muito Bom & $28(38,9)$ & $12(20)$ & $40(30,3)$ & \\
\hline & Excelência & $8(11,1)$ & - & $8(6,1)$ & \\
\hline \multirow{5}{*}{$\begin{array}{l}\text { FMS } \\
(\mathrm{cm})\end{array}$} & Fraco & $20(27,8)$ & $4(6,7)$ & $24(18,2)$ & \multirow{5}{*}{$<0,001^{*}$} \\
\hline & Razoável & $8(11,1)$ & $20(33,3)$ & $28(21,2)$ & \\
\hline & Bom & $24(33,3)$ & $16(26,7)$ & $40(30,3)$ & \\
\hline & Muito Bom & $16(22,2)$ & $20(33,3)$ & $36(27,3)$ & \\
\hline & Excelência & $4(5,6)$ & - & $4(3)$ & \\
\hline \multirow{4}{*}{$\begin{array}{l}\text { Agilidade } \\
\text { (seg.) }\end{array}$} & Fraco & $48(66,7)$ & $44(73,3)$ & $92(69,7)$ & \multirow{4}{*}{$0,016^{*}$} \\
\hline & Razoável & $8(11,1)$ & $8(13,3)$ & $16(12,1)$ & \\
\hline & Bom & $16(22,2)$ & $4(6,7)$ & $20(15,2)$ & \\
\hline & Muito Bom & - & $4(6,7)$ & $4(3)$ & \\
\hline \multirow{3}{*}{$\begin{array}{l}\text { Velocidade } \\
\text { (seg.) }\end{array}$} & Fraco & $32(44,4)$ & $32(53,3)$ & $64(48,5)$ & \multirow{3}{*}{$0,002^{*}$} \\
\hline & Razoável & $12(16,7)$ & $20(33,3)$ & $32(24,2)$ & \\
\hline & Bom & $28(38,9)$ & $8(13,3)$ & $36(27,3)$ & \\
\hline
\end{tabular}

Legendas: IMC: Índice de massa corporal; RCE: Relação cintura/estatura; FMI: Força explosiva de membros inferiores; FMS: Força explosiva de membros superiores; M: metros; Seg.: Segundos; Qtd.: Quantidade. Teste "Qui-quadrado" $(x 2)$. (* diferença $p<0,05)$.

Esse estudo buscou identificar e comparar o perfil da aptidão física em escolares praticantes de mini tênis. Os resultados indicam que os indivíduos apresentam aptidão física adequada para a idade e exibem bons desfechos em três de cinco indicadores de saúde (RCE, resistência geral e força/ resistência abdominal). Em relação ao desempenho motor, $60,6 \%$ da amostra, respectivamente, atendem aos critérios de desempenho nos indicadores de FMI e FMS. Contudo, na agilidade e velocidade, a maioria classifica-se com desempenho fraco.

As medidas antropométricas e de composição corporal têm sido exibidas como um fator importante para os critérios de desempenho em diversas modalidades esportivas (DJOKIĆ; MUNIVRANA; LEVAJAC, 2019). Nesse estudo, o IMC dos meninos e das meninas exibiram as proporções de $72,2 \%$ vs. $86,7 \%$, respectivamente, na zona de risco à saúde. Contudo, para a RCE observa-se que os $81,8 \%$ se classificam-se na zona saudá- 
vel, com maior disposição para o sexo feminino. Corroborando, outros estudos (MONTORO et al., 2016; SAGRILO JUNIOR et al. 2016) utilizaram o mesmo protocolo de testes dessa pesquisa, o PROESP (2016), e identificaram um percentual menor que $50 \%$ dos avaliados na zona de risco para o IMC.

Um baixo nível de gordura corporal é importante em tenistas, para alcançarem bons resultados de desempenho motor (agilidade e velocidade) (SCHLUGA, 2016). Kovacs (2006) destaca que as variáveis de velocidade, agilidade e força são imprescindíveis para quem pratica o tênis de campo. Assim, tenistas necessitam de uma preparação eficiente nesses indicadores predominantes (DURIGAN et al., 2013). Fato esse, encontrado somente nas variáveis de força, dos escolares desse estudo.

O perfil de aptidão física relacionado ao desempenho motor nos indicadores de agilidade e velocidade dos praticantes de mini tênis dessa pesquisa, identificam-se como fracos. Ainda, ao agrupar as categorias "fraco" e "razoável" de cada indicador, se obtém um percentual de $81,8 \%$ (agilidade) e $72,5 \%$ (velocidade) de indivíduos que não atenderam aos critérios de desempenho. Similarmente, Souza et al. (2017) avaliaram 10 meninos praticantes de tênis de campo e encontraram desempenho fraco para velocidade, agilidade e FMI. Mello et al. (2015) também evidenciam que, quando consideramos os testes de agilidade e velocidade, a grande maioria das crianças encontram-se com resultados entre razoável e fraco, sendo que nenhuma delas conseguiu atingir o desempenho excelente.

Quando levamos em consideração os testes de FMI e FMS, a maioria dos escolares praticantes de mini tênis, dispõem de desempenho entre "bom", "muito bom" e "excelência", diferentemente do encontrado por Mello et al. (2015). Em estudo similar (SCHUBERT et al., 2016), a proporção de sujeitos que atingiram os critérios de saúde foi de aproximadamente $55 \%$ nos esportes coletivos e pouco mais de $60 \%$ para força nos esportes individuais. No tênis, a força muscular é indispensável para o tenista deslocar-se rapidamente e para rebater a bola. A FMI é essencial para o atleta desempenhar uma boa potência de aceleração. Dessa maneira, a força rápida permite a execução de ações por um longo período, sem que o indivíduo perca sua velocidade de execução de sua ação motora (MAHN; GAVIÃO, 2010).

Em relação a flexibilidade, para tenistas ela interfere, principalmente, nos músculos de membros inferiores, uma vez que, exige amplitude máxima para não sofrer interferência no rendimento sobre o estado de encurtamento. Nesse estudo, a maioria dos escolares foram classificadas na zona de risco à saúde, ou seja, não são flexíveis o suficiente para atender aos critérios de saúde nesse teste. Todavia, há uma tendência maior de meninas na zona de saudável (72,2\%). Godoi Filho e Farias (2015) corroboram, afirmando que maior parte da amostra estudada apresentou índices de flexibilidade que não atendiam o mínimo exigido para a condição de saúde. Resultados positivos para meninas também foram encontrados em outras pesquisas (MASCARENHAS et al., 2013; DORNELES et al., 2016).

A resistência muscular é outro fator importante no treino de tênis. Um tenista deve ser capaz de aguentar várias horas e bater centenas de golpes num só jogo. Quanto mais músculos conseguir contrair sem atingir a fadiga, maior é a resistência muscular. Os escolares dessa pesquisa apresentaram desempenho satisfatório no teste de força/resistência abdominal, a maioria estão na zona de saudável de acordo com o protocolo de avaliação. Estes resultados não vão ao encontro dos achados de Pelegrini et al. (2011), o qual, mais de $50 \%$ dos escolares foram classificados na zona de risco. Em outro estudo (LUZ et al., 2008), com crianças e adolescentes de um projeto de iniciação ao tênis de campo, meninos realizaram 34 movimentos vs. 33 movimentos das meninas, em 1 minuto, ou seja, o desempenho no teste de resistência muscular localizada não foi significativo entre os sexos.

Ao comparar os desempenhos no teste de força/resistência abdominal em função do sexo, nota-se que houve maiores ocorrências de meninas na zona saudável. Este quadro pode acontecer pelo fato de que elas também apresentaram melhor desempenho no teste de flexibilidade, ou ainda pelo tipo de atividades físicas e motoras que estão engajadas. Esses resultados podem se justificar pelo fato de que a força abdominal é um fator determinante no desempenho do teste sentar-e-alcançar, uma vez que músculos abdominais fortes influenciam em um maior grau de flexão (MALINA; BOUCHARD; BAR-OR, 2009).

Com relação a resistência geral, contata-se resultados satisfatórios entre meninos e meninas, $81,8 \%$ se destacam na zona saudável, não apresentando diferença entre o sexo. Em contrapartida, outros estudos encontraram dados insatisfatórios para essa condição cardiorrespiratória (CONTREIRA et al., 2016; HENKES et al., 2013). Diversos fatores podem ser associados ao desempenho na aptidão cardiorrespiratória, entre eles a fase do estirão de crescimento da adolescência, a diferença entre os sexos na competência de práticas de atividades, média corporal e o estilo de vida sedentário, que em qualquer idade leva a diminuição da capacidade cardíaca, respiratório e vascular (HAYWOOD; GETCHELL, 2016).

Vale ressaltar que uma possível limitação deste estudo está no fato de considerar apenas as diferenças entre os sexos, tendo em vista que a comparação com outras variáveis poderia favorecer o entendimento da influência de cada uma delas na aptidão física dos praticantes de mini tênis. Outra possível limitação, está na objeção em avaliar o estágio maturacional da amostra. Este tipo de avaliação pode, frequentemente, causar constrangimento ao avaliado, por ter que apontar seu órgão genital através de uma figura ou desenho e/ou por se colocar seminu diante do observador médico para tal avaliação, ao mesmo tempo causando desconforto ao avaliador (MARTIN et al., 2001). Corroborando, alguns autores (MALINA; BOUCHARD, 2002; GALLAHUE; OZMUN; GOODWAY, 2013) ao observarem as variáveis de crescimento, composição corporal e desempenho motor, advogam que, na faixa etária que compreende dos 6 aos 12 anos de idade, as diferenças entre os sexos existem, entretanto não se mostram significativas. Malina, Bouchard e Bar-Or (2009) e Rogol, Roemmich e Clark (2002), também afirmam que as diferenças no desempenho motor entre meninos e meninas é pequena e inexistente, desde que ambos tenham oportunidade de prática motora.

Apesar destas limitações, realizou-se uma pesquisa com um tema pouco explorado entre os pesquisadores, que é o perfil da aptidão física de praticantes de mini tênis, assim, este estudo torna-se um importante canal de informações que servirá como subsídio para que profissionais da modalidade possam planejar ações direcionadas ao controle da carga externa de treino dos seus alunos e/ou atletas de maneira mais sistematizada.

\section{CONCLUSÃO}

Com o objetivo de identificar e comparar o perfil da aptidão física dos praticantes de mini tênis, este estudo encontrou diferenças entre os sexos nas variáveis de flexibilidade e força-resistência abdominal, meninas são mais flexíveis e estão na 
zona saudável à saúde, em relação a seus pares. Para os outros indicadores, os resultados exibiram semelhanças, em geral, foram classificados com o IMC na zona de risco à saúde, resistência geral na zona saudável, FMI e FMS atendem aos critérios de desempenho, agilidade fraca e velocidade entre fraca e razoável. Dessa maneira, após um ano de prática de mini tênis, a aptidão física dos escolares avaliados está abaixo do esperado, visto que, o IMC está elevado para a idade e o desempenho nos testes de agilidade e velocidade (essenciais para o mini tênis) não foi positivo.

\section{REFERÊNCIAS}

BRUSTOLIN, M. Tênis no Brasil: história, ensino e ideias. Rio de Janeiro: Editora Sprint, 1995.

CHIMINAZZO, J. G. C.; AZZI, R. G. Atividades competitivas e "esgotamento" (burnout) no tênis de campo infanto-juvenil. Tênis de campo: temas em debate. Taubaté: Cabral, p. 71-84, 2005.

CONTREIRA, A. R.; PIZZO, G. C; ROCHA, F. F. d.; LAZIER-LEÃO, T. R.; CARUZZO, A. M.; COPETTI, F.; BELTRAME, T. S. Perfil de aptidão física relacionada a saúde em adolescentes. Saúde e Pesquisa, Maringá, v. 9, n. 2, p. 309-315, 2016.

COSTA, N. L. da. Mini-Tênis na Educação Física Escolar. 2019. 49f. TCC (Graduação em Educação Física) - Universidade Federal de Santa Catarina, Florianópolis, 2019.

DJOKIĆ, Z.; MUNIVRANA, G.; LEVAJAC, D. Anthropometric characteristics of top-class World and European male table tennis players. EUROPE, [s.I.], v. 44, n. 1, p. 170-5, 2019

DORNELES, R. C. G.; OLIVEIRA, H. L. R.; BERGMANN, M. L. de A.; BERGMANN, G. G. Flexibility and muscle strength/resistance indicators and screening of low back pain in adolescents. Revista Brasileira de Cineantropometria \& Desempenho Humano, Florianópolis, v. 18, n. 1, p. 93-102, 2016.

DUMITH, S. C.; RAMIRES, V. V.; SOUZA, M. J. A.; MORAES, D. S.; PETRY, F. G.; OLIVEIRA, E. S.; ... ; MARQUES, A. C. Aptidão física relacionada ao desempenho motor em escolares de sete a 15 anos. Revista Brasileira de Educação Física e Esporte, São Paulo, v. 24, n. 1, p. 5-14, 2010.

DURIGAN, J. Z.; DOURADO, A. C.; SANTOS, A. H. dos; CARVALHO, V. A. Q.; RAMOS, M.; STANGANELLI, L. C. R. Efeitos do treinamento pliométrico sobre a potência de membros inferiores e a velocidade em tenistas da categoria juvenil. Revista da Educação Física, Maringá, v. 24, n. 4, p. 617-26, 2013.

GALLAHUE, D. L.; OZMUN, J. C.; GOODWAY, J. D. Compreendendo o desenvolvimento motor: bebês, crianças, adolescentes e adultos. Porto Alegre: AMGH, 2013.

GAYA, A. C. A.; SILVA, G. M. G. de; CARDOSO, M. F. de S.; CARDOSO, L. T. Talento esportivo: estudo de indicadores somatomotores na seleção para o desporto de excelência. Revista Perfil, Porto Alegre, v. 6, n. 6, p. 86-96, 2002.

GODOI FILHO, J. R. de M.; FARIAS, E. da S. Aptidão física de escolares do sudoeste da Amazônia Ocidental em diferentes estágios de maturação sexual. Revista Brasileira de Educação Física e Esporte, São Paulo, v. 29, n. 4, p. 631-9, 2015

HAYWOOD, K. M.; GETCHELL, N. Desenvolvimento Motor ao Longo da Vida. 6. ed. Porto Alegre: Artmed, 2016.

HENKES, C. M.; BORFE, L.; MURADÁS, R.; TORNQUIST, L.; BURGOS, M. S. Aptidão física relacionada ao desempenho de escolares: estudo comparativo dos hemisférios norte-sul-leste-oeste da zona rural de Santa Cruz do Sul RS. Cinergis, Santa Cruz do Sul, v. 14, n. 4, p. 206-209, 2013.

ITF. International Tennis Federation. Minitênis. 1993. Disponível em: <http://www.tennisplayandstay.com/\#Progression\&gt> Acessado em: 08 de junho de 2020.

KOVACS, M. S. Applied physiology of tennis performance. British Journal of Sports Medicine, Loughborough, v. 40, n. 5, p. 381-6, 2006.
LUZ, M. L. P. da; FILLIPIN, L.; ZACOUTEGUY, F.; LUIZ, G.; DE PAULA, P. A.; SOUZA, G. da $S$. Indicadores de níveis de aptidão física em crianças e adolescentes de um projeto de iniciação ao tênis de campo. Lecturas: Educación Física y Deportes, Revista Digital, Buenos Aires, v. 13, n. 123, 2008. Disponível em: <https://www.efdeportes.com/efd123/niveis-deaptidao-fisica-em-criancas-e-adolescentes-tenis-de-campo.htm>. Acessado em: 13 de abril de 2020.

MAHN, P. A.; GAVIÃO, M. B. A influência do treinamento resistido em atletas de tênis de campo. Revista Brasileira de Ciências da Saúde, João Pessoa, v. 8, n. 24, p. 3-7, 2010.

MALINA, R. M.; BOUCHARD, C. Atividade física do atleta jovem: do crescimento à maturação. São Paulo: Roca, 2002.

MALINA, R. M.; BOUCHARD, C.; BAR-OR, O. Crescimento, maturação e atividade física. São Paulo: Phorte, 2009.

MARTIN, R. H. C.; UEZU, R.; PARRA, S. A.; ARENA, S. S.; BOJIKIAN, L. P.; BOHME, M. T. S. Auto-avaliação da maturação sexual masculina por meio da utilização de desenhos e fotos. Revista Paulista de Educação Física, São Paulo, v. 15, n. 2, p. 212-22, 2001.

MASCARENHAS, L. P. G.; FERREIRA, A. B.; LIMA, V. d. A. d. L.; GRZELCZAK, M.T. Estudo comparativo da aptidão física entre crianças de escola pública e particular: uma visão regional. Cinergis, Santa Cruz do Sul, v. 14, n. 3, p. 157-160, 2013.

MELLO, J. B.; HERNANDEZ, M. d. S.; FARIAS, V. M.; PINHEIRO, E. d. S.; BERGMANN, G. G. Aptidão física relacionada ao desempenho motor de adolescentes de Uruguaiana, Rio Grande do Sul. Revista Brasileira de Ciência e Movimento, São Paulo, v. 23, n. 4, p. 72-9, 2015.

MONTORO, A. P. P. N.; LEITE, C. R.; ESPÍNDOLA, J. A.; ALEXANDRE, J. M.; REIS, M. d. S.; CAPISTRANO, R.; ... ; BELTRAME, T. S. Aptidão física relacionada à saúde de escolares com idade de 7 a 10 anos. ABCS Health Sciences, Santo André, v. 41, n. 1, p. 29-36, 2016.

PELEGRINI, A.; SILVA, D. A. S.; PETROSKI, E. L.; GLANER, M. F. Aptidão física relacionada à saúde de escolares brasileiros: dados do projeto esporte Brasil. Revista Brasileira de Medicina do Esporte, São Paulo, v. 17, n. 2, p. 92-6, 2011.

PROESP. Projeto Esporte Brasil. Manual. Porto Alegre. 2016. Disponível em: <https://www.proesp.ufrgs.br> Acesso em: 16 de janeiro de 2020.

ROGOL, A. D.; ROEMMICH, J. N.; CLARK, P. A. Growth at puberty. Journal of Adolescent Health, New York, v. 31, n. 6, p. 192-200, 2002.

SCHLUGA FILHO, J. L.; RIBAS, M. R.; NOGUEIRA, L. d. O.; ANDRADE JÚNIOR, C. d.; FERNANDES, P.; BASSAN, J. C. Motor and morphological profile of tennis players from 11 to 15 years old. Revista Andaluza de Medicina del Deporte, Barcelona, v. 9, n. 3, p. 114-8, 2016.

SAGRILO JUNIOR, C.; REIS, G. R. d.; JAEGER, D. B.; SIQUEIRA, O.D.; CRESCENTE, L. A.; GARLIPP, D. C. Associação entre o sobrepeso e hipertensão arterial em crianças e adolescentes. Cinergis, Santa Cruz do Sul, v. 17, n. 2, p. 125-8, 2016.

SCHLUGA FILHO, J. L.; RIBAS, M. R.; NOGUEIRA, L. d. O.; ANDRADE JUNIOR, C. d.; FERNANDES, P.; BASSAN, J. C. Perfil motor y morfológico de jugadores de tenis de 11 a 15 años de edad. Revista Andaluza de Medicina del Deporte, Barcelona, v. 9, n. 3, p. 114-8, 2016.

SCHUBERT, A.; JANUÁRIO, R. S. B.; CASONATTO, J. SONOO, C. N. Aptidão física relacionada à prática esportiva em crianças e adolescentes. Revista Brasileira de Medicina do Esporte, São Paulo, v. 22, n. 2, p. 142-6, 2016.

SILVA, T. R.; CONTENÇAS, T. S.; MARQUES, A. Avaliação do desenvolvimento motor em crianças praticantes e não praticantes de exercícios físicos. Revista Brasileira de Fisiologia do Exercício, São Paulo, v. 16, n. 4, p. 208-14, 2017.

SILVA, V. C.; TEIXEIRA, F. A. A.; ALVES, F. R.; SOUZA, E. A. de. Análise de aptidão física de adolescentes praticantes de futsal. Revista Brasileira de Futsal e Futebol, São Paulo, v. 9, n. 34, p. 250-7, 2017.

SOUZA, W. C. d.; BONETTE, A.; SMOLAREK, A. d. C.; SOUZA JUNIOR, T. P. d.; MASCARENHAS, L. P. G. Avaliação da velocidade, agilidade e força de membros inferiores de adolescentes praticantes de tênis de campo. VIREF Revista de Educación Física, Medellin, v. 6, n. 3, p. 1-8, 2017. 
VENÂNCIO, P. E. M.; SILVA, J. P. d. S.; RIBEIRO, H. L.; SOARES, V.; SANTANA, F.; TOLENTINO, G. P. Aptidão física em adolescentes praticantes de futebol. Revista Brasileira de Futsal e Futebol, São Paulo, v. 10, n. 36, p. 41-8, 2018.

\section{CONFLITO DE INTERESSE}

Os autores do estudo declaram não haver conflito de interesses.

\section{FINANCIAMENTO}

Este estudo não recebeu nenhum tipo de apoio financeiro.

\section{ORCID E E-MAIL DOS AUTORES}

Flávia Évelin Bandeira Lima (Autor Correspondente) ORCID: 0000-0002-7026-3354.

E-mail: flavia.lima@uenp.edu.br

Walcir Ferreira Lima

ORCID: 0000-0003-0932-7969.

E-mail: walcirflima@uenp.edu.br

Mariane Aparecida Coco

ORCID: 0000-0002-5995-7363.

E-mail: mariuenpedf@gmail.com

Fellipe Bandeira Lima

ORCID: 0000-0001-5917-6412.

E-mail: lima_fisioterapia@hotmail.com

Silvia Bandeira da Silva Lima

ORCID: 0000-0002-6862-2944.

E-mail: silviabslima@uenp.edu.br 